\title{
Criminologie
}

\section{Le mégacrime, légitimité, légalité et obéissance}

\section{Stéphane Leman-Langlois}

Volume 39, numéro 2, automne 2006

Le crime de génocide : Construction d'un paradigme pour la criminologie, la philosophie et le droit pénal

URI : https://id.erudit.org/iderudit/014426ar

DOI : https://doi.org/10.7202/014426ar

Aller au sommaire du numéro

Éditeur(s)

Les Presses de l'Université de Montréal

ISSN

0316-0041 (imprimé)

1492-1367 (numérique)

Découvrir la revue

Citer cet article

Leman-Langlois, S. (2006). Le mégacrime, légitimité, légalité et obéissance. Criminologie, 39(2), 23-37. https://doi.org/10.7202/014426ar

\section{Résumé de l'article}

Cet article passe en revue diverses théories criminologiques et les confronte à la réalité de ce que l'auteur appelle les « mégacrimes » (actes généralement criminalisés et produisant la perte de vies humaines à grande échelle). Il en ressort que les outils théoriques de la criminologie sont largement impuissants lorsqu'il s'agit de rendre compte de tels crimes. Non pas qu'ils soient à l'extérieur de son objet, il s'agit bien de conduites dommageables réfléchies, organisées, produisant des victimes et impliquant des systèmes de droit, après tout, mais bien que la discipline se soit surtout penchée sur les conduites purement individuelles.
Ce document est protégé par la loi sur le droit d'auteur. L'utilisation des services d'Érudit (y compris la reproduction) est assujettie à sa politique d'utilisation que vous pouvez consulter en ligne.

https://apropos.erudit.org/fr/usagers/politique-dutilisation/ 


\title{
Le mégacrime, légitimité, légalité et obéissance
}

\author{
Stéphane Leman-Langlois \\ Centre international de criminologie comparée \\ s.langlois@umontreal.ca
}

RÉSUMÉ - Cet article passe en revue diverses théories criminologiques et les confronte à la réalité de ce que l'auteur appelle les «mégacrimes» (actes généralement criminalisés et produisant la perte de vies humaines à grande échelle). Il en ressort que les outils théoriques de la criminologie sont largement impuissants lorsqu'il s'agit de rendre compte de tels crimes. Non pas qu'ils soient à l'extérieur de son objet, il s'agit bien de conduites dommageables réfléchies, organisées, produisant des victimes et impliquant des systèmes de droit, après tout, mais bien que la discipline se soit surtout penchée sur les conduites purement individuelles.

\begin{abstract}
This paper reviews a few current criminological theories and evaluates their ability to account for what the author refers to as "mega-crimes" (acts widely criminalized at the international level and producing large-scale loss of human life). The exercise shows that criminology is simply incapable of explaining mega-crimes. Not that these acts are outside of its defined field of study: after all they, like common crimes, cause damage to victims, are voluntary, thought-out actions, organized and in interaction with various judicial systems. The reason for this incapability to account for vast portions of what should be its object is rather to be found in its narrow but persistent focus on purely individual conduct.
\end{abstract}

\section{Introduction}

La criminologie, force est de le constater, voit peu au-delà des préoccupations sécuritaires immédiates des classes moyennes des démocraties occidentales. Un marxien dirait qu'elle reste la science des menaces perçues par le consommateur aisé, à son confort quotidien. Ainsi, les notions de crime de guerre, de crime contre l'bumanité, de crime contre la paix (s'il faut suivre les catégories de Nuremberg) et de crime de l'État 
en général lui échappent entièrement. Bien qu'elle soit à la mode, la question du terrorisme est également peu couverte par la recherche criminologique. En général ces questions ont été laissées aux psychologues pour le niveau micro et aux politicologues pour le niveau macro.

Pourtant, les grands crimes ou mégacrimes comme certains les ont appelés ne sont pas sans répercussions sur nos sociétés plus calmes. Exterminations (par les armes, par les famines, par les déportations dans des régions difficiles), terrorisme de masse, répression violente à grande échelle, nettoyages ethniques, viols de masse, mutilations de masse, guerres menées contre des civils, etc., leurs conséquences finissent toujours par nous toucher, entre autres par l'entremise de l'immigration, de l'aide internationale ou de la sécurité des Canadiens qui voyagent à l'étranger.

Ces conséquences bien tangibles, par contre, ne devraient pas convaincre le criminologue toujours à la recherche de vrais crimes, objectifs et reconnaissables in re, que son Saint-Graal est enfin à portée de «lèvres». Bien qu'il soit expéditif dans le cadre de ce texte de qualifier certains comportements de mégacrimes, la catégorie est peut-être encore plus problématique que celle du crime commun. Une chose est certaine, dans la plupart des exemples de ces comportements, le criminologue n'a plus aucun repli sur les définitions légales et sur les sanctions établies pour définir les actes comme crimes (voir Brodeur et Ouellet, 2005). Tous les mégacrimes sont des constructions juridiques avant tout, et cet aspect ne devrait pas échapper au chercheur qui s'y intéresse.

Dans ce qui suit, nous verrons à la suite trois angles d'exploration possibles des mégacrimes, chacun selon un paradigme théorique différent. Le premier consiste à appliquer l'idée classique de recherche des bénéfices et de fuite des souffrances comme motivation de l'agir des acteurs. Dans cette conception du crime, l'individu saisit les opportunités criminelles parce qu'une évaluation des conséquences mène à cette conclusion. Dans la deuxième, nous examinerons la dynamique de groupe qui s'installe dans une organisation vouée à l'extermination d'êtres humains. En dernier lieu, nous nous pencherons sur la thèse, populaire auprès du Tribunal pénal international pour le Rwanda, voulant que les médias puissent causer certains crimes graves par l'information qu'ils diffusent. 


\section{Ordres, rationalités et stratégies}

La pénologie classique, née et raffinée au temps des Lumières avec Montesquieu, Beccaria et Bentham, situa l'agir humain au centre d'un univers économique fondé sur la maximisation des bénéfices individuels. Dans cet univers, l'acteur agit en fonction de la satisfaction approximative de ses désirs à plus ou moins long terme (cette fourchette temporelle laissant place à certaines différences psychologiques). Si certains de ses actes se trouvent à être des crimes, c'est que ni l'État ni l'environnement social n'ont réussi à en dissuader les auteurs en faisant pencher la balance des coûts sous le poids des sanctions officielles ou informelles. Cette conception de la criminalité semble naturellement aller de pair avec les crimes visant l'acquisition de biens ou d'argent (entre autres, la criminalité organisée), mais certains auteurs (le cas le plus classique est Hirshi et Gottfredson, 1994; voir aussi Cusson, 2005) ont tenté de démontrer que tout crime, comme toute action humaine, n'est jamais que l'aboutissement d'un calcul économique.

Comme tout bon fonctionnaire allemand, Adolph Eichmann cherchait surtout l'avancement au sein d'une organisation bureaucratique: celle-ci se trouvant être la Gestapo. Sa participation aux objectifs généraux de l'institution prit la tournure qu'on connaît. Un parallèle pourrait être dressé entre les actes d'Eichmann et un vol qualifié. On peut appliquer au vol qualifié en général une logique essentiellement économique: si le voleur commet une quelconque violence, cette violence reste toujours un simple outil visant à assurer le succès de l'opération économique qui motive le tout. Dans ce parallèle, Eichmann s'adonne à certains actes (indirectement) violents afin de mousser sa carrière personnelle et d'assurer son avancement dans l'organisation (avantages sociaux, salaire, grade, médailles, etc.). On le voit, la comparaison souffre de plusieurs problèmes: premièrement, le concept d'opportunité semble mal s'appliquer parce que l'avancement reste un concept relativement flou et assez loin du concret, Eichmann d'ailleurs ne gravit que peu les échelons de sa hiérarchie et se plaint que l'expertise, trop spécialisée, qu'il avait apportée en déportant des millions de victimes le cantonnait dans des emplois subalternes. Deuxièmement, l'avancement dans la Gestapo et dans le Bureau central de sécurité du Reich (RSHA) ne nécessitait pas une coopération au génocide. Troisièmement, il semble y avoir une certaine disproportion entre ce que l'individu ordinaire est prêt à faire afin d'obtenir de l'avancement 
et les quelque 2 millions de victimes que fit Eichmann au cours de sa carrière.

Lautre facette de cette rationalité économique consiste à fuir la sanction; les participants auraient en quelque sorte été poussés à agir de la sorte sous la menace de leur état-major (dont la rationalité resterait ainsi à établir). Or il est désormais démontré que les nazis se désistant du projet hitlérien n'encouraient aucune sanction (voir Browning, 1992; Israël, 1992; Goldhagen, 1996).

Enfin, il faut explorer la possibilité qu'un mégacrime ne soit en fait qu'une opportunité généralisée d'assouvir des désirs (ataviques, dirait sans doute Lombroso) toujours présents chez l'individu, mais généralement réprimés par le droit ou par d'autres contrôles sociaux. Alternativement, le mégacrime pourrait être une autre forme de retour à l'enfance et à la peur hobbesienne de la mort violente, menant aux pires excès, sorte de généralisation de la mentalité des foules (Buford, 1991). Dans une perspective classique, cette dernière thèse semble obliger à une navigation impossible entre le Charybde d'une rationalité des instincts, qui rendrait le concept de rationalité superflu (ce dont s'approcha tout de même Bentham), et le Scylla du droit tout puissant réprimant parfaitement les bas instincts du peuple.

À l'autre extrémité du spectre génocidaire se trouve le massacre des Tutsis du Rwanda en 1994 (Gourevitch, 1998; Hatzfeld, 2003). Si la destruction de la communauté juive d'Europe se fit à renforts de technologies industrielles et bureaucratiques puissantes (Bauman, 1989), celle des Tutsis est beaucoup plus proche des flambées racistes de l'Europe des pogroms préhitlériens ou des massacres entre voisins dans l'ex-Yougoslavie. Bien sûr, aucun de ces cas de figure n'est une illustration parfaite de sa catégorie: au Rwanda comme en Yougoslavie, une organisation de base a facilité les choses (Hagan, 2003); les exécutants du III ${ }^{\text {e }}$ Reich n'étaient pas tous des technologues froids et distants (Israël, 1992). Néanmoins, durant le désastre rwandais plusieurs attaquants profitèrent du chaos pour s'approprier les terres et les biens de leurs victimes. D'autres étaient menacés de mort s'ils ne participaient pas aux massacres. Aucune de ces deux raisons rationnelles ne permet pourtant de rendre compte de l'ampleur du mégacrime en question.

S'il est aujourd'hui bien difficile d'ignorer ce que Popper (1985) appela le principe de rationalité, axiome décrivant l'action humaine comme intrinsèquement rationnelle, il s'agit d'une rationalité localisée dans un contexte culturel, limitée par les facultés de l'acteur et par les 
actions des autres (Crozier et Freidberg, 1977). Ainsi, l'acteur rationnel évolue dans une structure sociopolitique déterminée, qu'il évalue et tente de comprendre avec des outils conceptuels définis et provenant de la culture locale. Dans bien des cas, le constat que l'acteur impliqué dans un mégacrime est rationnel s'est opposé aux perspectives pathologisantes qui voyaient, par exemple, tous les nazis comme psychopathes ou sadiques et tout terroriste comme un illuminé abandonné par sa raison (entre autres, Merari, 1990; Manoni, 2004). Au sens strict où il 1) choisit certaines actions, 2) en visant un but et 3) dans un contexte, l'acteur du mégacrime est rationnel, par définition. Néanmoins, ceci aide peu à comprendre à quoi, et comment il réfléchit, et encore moins pourquoi il choisit certains moyens plutôt que d'autres.

\section{Contextes, organisations, bureaucraties}

Hannah Arendt, dans son ouvrage célèbre sur l'État totalitaire (Arendt, 1951), avait fait un premier inventaire de l'environnement social qui permet la dérive vers une criminalité de grande envergure. Mis à l'épreuve lors du procès historique d'Adolph Eichman (Arendt, 1963), son modèle semble le mieux expliquer comment un bureaucrate allemand parfaitement ordinaire a pu devenir un spécialiste de la déportation vers les camps d'extermination. Selon elle, c'est le contexte social de l'individu qui encourage sa participation à un programme large d'action impliquant également ses pairs, et non une simple hiérarchie coercitive. Comme bien d'autres membres de la Gestapo, Eichmann n'était pas exceptionnel pour sa haine des Juifs ou son amour pour le Führer. Autrement dit, son passé semble exclure la thèse voulant que l'individu voulant prendre part à certaines activités, criminelles ou non, cherche à se joindre à des pairs ayant les mêmes objectifs et caractéristiques sociopsychologiques. Le rôle de l'État nazi, par ailleurs, n'était pas de faire plier des milliers de participants récalcitrants, mais bien de créer un monde où l'extermination était possible. Pour Arendt, Eichmann s'est retrouvé dans un contexte totalitaire, non pas au sens où les ordres étaient impératifs, mais plutôt parce que la pensée alternative devenait de plus en plus difficile. Au point où la caractéristique primordiale d'un crime, celle qui en fait une tentation interdite, était renversée: c'était au contraire le désistement du projet nazi qui restait au stade de la tentation inassouvie. Ajoutons au passage que l'idée de bénéfice personnel, si 
chère aux classiques, a été également un facteur ajoutant au flou moral: Eichmann se révoltait toujours à l'accusation qu'il aurait soutiré des diamants aux Juifs qu'il déportait, ce qui lui permettait de revendiquer une probité sans faille.

Ainsi, le phénomène ressemble davantage à celui décrit par Sutherland et Cressey (1966) comme une association différentielle (plus récemment par Akers, 1998), où chaque individu se liant au groupe apprend peu à peu à voir la réalité d'une certaine façon. Ceci correspond à l'isolement progressif des membres des SS et de leur intégration de plus en plus exclusive au sein du groupe, décrite par Arendt. À un tout autre sujet, Sageman (2004) a décrit de manière similaire l'adoption et la radicalisation de recrues dans les réseaux terroristes jihadistes. Waller (2002), malgré une aussi courte qu'infructueuse dérive dans le champ de la sociologie évolutive (comme Brannigan, 1998 ou Ghigilieri, 1999), offre une vue plus systématisée du mécanisme. En particulier, la participation à des actions précédemment jugées immorales requiert que l'acteur soit situé dans un système de croyances relativement particulières, surtout celles qui favorisent l'obéissance aux ordres et la déférence pour l'autorité en général. Cette croyance se forge de façon beaucoup plus efficace dans le milieu fermé d'une bureaucratie totalitaire, relativement isolée du monde extérieur. C'est également la dynamique sociopsychologique qui se propage dans les sectes extrémistes (Casoni et Brunet, 2003). Particulièrement utile ici est l'approche de la formation et du maintien de l'identité sociale (Jenkins, 1996), qui écarte la différenciation artificielle entre l'individu et le groupe. Le criminologue y reconnaîtra également les techniques de neutralisation observées par Matza (1964), qui permettent aux membres d'un groupe de penser la moralité de leurs actions en dehors de la moralité générale abstraite à laquelle ils continuent de souscrire.

Plusieurs études ont conclu que les ordres et l'obéissance étaient les facteurs principaux de mégacrimes, à commencer par celle de la personnalité autoritaire d'Adorno (1969). Plus récemment, les psychologues sociaux, Kelman et Hamilton (1989), ont également touché à ce qu'ils ont appelé les «massacres sanctionnés» (sanctioned massacres), dont les auteurs ne sont plus des acteurs, mais bien des agents manipulés par des donneurs d'ordres. Le premier problème ici est de conceptualiser le mégacrime comme conçu par des personnes qui ne le commettent pas, mais qui ordonnent aux autres de le faire sans avoir à le concevoir, ce qui équivaut à contourner la question. De plus, dans les faits, cette 
séparation entre les dirigeants (rationnels) et les exécutants (manipulés) reste en général plutôt artificielle. Au contraire, Pape (2005) montre bien combien à la fois les exécutants et les dirigeants sont animés de motifs rationnels, bien que différents. Pape minimise toutefois beaucoup trop l'influence du contexte culturel sur l'acteur, rejetant, par exemple, tout effet de l'endoctrinement des suicidés à l'aide de références à l'Islam et fondant ses conclusions sur quelques cas trop bien choisis. En s'opposant (avec raison) à ceux qui trouvent en l'Islam la cause finale $\mathrm{du}$ terrorisme jihadiste, il pèche par excès contraire en ignorant volontairement que non seulement les objectifs, mais surtout les stratégies choisies par les extrémistes sont contingents, dépendant de plusieurs facteurs culturels et contextuels. Tout comme le génocide, le terrorisme suicidaire n'est pas la seule conclusion possible dans la réalisation du but politique des insurgés; le choix de tactiques reste donc tout entier à expliquer. Autrement dit, le fait qu'on puisse placer une intention rationnelle derrière un mégacrime ne suffit pas à établir un lien entre cette intention et la tactique employée. Cette approche ne permet aucune prédiction.

Autre problème de l'autorité, comme l'ont montré Breton et Wintrobe (1982; 1986), Goldhagen (1996) et Browning (1992): pour s'étendre, le mégacrime ne nécessite tout simplement pas que des ordres soient donnés. Dans l'analyse de la structure bureaucratique du Reich que font Breton et Wintrobe, le lecteur est confronté à l'absence relative d'ordre et de directive. On y voit plutôt des «appels d'offres» et une compétition entre les bureaucrates pour résoudre les problèmes identifiés. Si certains crimes sont sans doute des crimes d'autorité, comme plusieurs crimes de guerre, la conclusion que l'être humain normal ne commet des atrocités à grande échelle que sous les ordres d'autres est clairement intenable. Il faut repenser la notion d'autorité pour y inclure une idée plus vague d'autorisation: ce ne sont pas les ordres en eux-mêmes qui sont nécessaires, mais bien un contexte social, moral et légal qui encourage et, bien sûr, permet les actes reprochés.

Enfin, dernier problème de l'explication par l'autorité, on doit pour l'accepter supposer que les acteurs croient, en effet, que leur action est immorale, ce qui n'est généralement pas le cas. Plusieurs études ont montré de façon définitive que les auteurs de mégacrimes, les terroristes, par exemple, sont parfaitement convaincus de la moralité de leur décision. Leur décision de participer est déjà prise lorsqu'ils reçoivent leurs ordres. Bref, le crime autorisé est le résultat de jeux de pouvoir au 
sein d'un groupe où s'est installée une culture de neutralisation de certains actes communément considérés comme des crimes (immoraux et punissables) par les membres.

En pensant la rationalité de façon plus flexible, on peut intégrer les tactiques extrêmement violentes employées par les acteurs dans un modèle certes plus complexe, et sans doute moins élégant, mais qui a l'avantage de ne pas introduire de discontinuité dans l'explication. Merton et d'autres théoriciens de la tradition de la tension ont déjà montré que le comportement délinquant pouvait trouver sa source dans une inadéquation des objectifs personnels culturellement célébrés avec l'accès à des moyens socialement acceptables de les réaliser. Le mégacrime, sous cet angle, pourrait être un moyen peu apprécié, mais considéré par ses utilisateurs comme le seul outil disponible pour atteindre un but qu'ils considèrent absolument vital. $\mathrm{Si}$, dans la société de consommation glorifiant le succès financier, le vol, l'extorsion et les commerces illégaux se multiplient chez ceux qui n'ont pas le capital social voulu pour s'enrichir légalement, dans une société où la nation est menacée d'un cataclysme imminent, il paraît peut-être nécessaire de commettre un mégacrime pour la protéger. Pour utiliser un langage foucaldien, l'élément manquant est un discours qui crée une moralité et un espace social alternatifs, qui identifie le cataclysme en question et qui pousse vers une solution radicale. Malheureusement pour Merton, on ne peut supposer que ce discours sera le fruit d'un consensus large, et peut être localisé dans un groupe social restreint.

\section{Discours, propagande et participation}

Ainsi, au-delà du simple jugement de valeur, la perspective répandue voulant que la moralité des génocidaires, par exemple, soit suspendue ou renversée (par exemple, Waller, 2002) est peu satisfaisante et encombrée d'un concept peu scientifique de moralité absolue ou du moins d'une échelle universelle de moralité qui n'est observable nulle part. En fait, il est plus utile de concevoir le mégacrime non pas comme immoral mais bien comme excessivement moral. C'est le déploiement d'un discours moral absolutiste qui sert de justification et de rationalisation aux actes extraordinaires, tout comme les discours moraux ordinaires sont des justifications aux actes ordinaires (Spector et Kitsuse, 2001 : 85-95).

Julius Streicher, éditeur du journal ultraraciste Der Stürmer entre 1923 et 1945, a été condamné à mort et pendu à Nuremberg (non pas pour 
avoir participé au génocide, mais pour avoir persécuté les Juifs par l'entremise de son journal haineux, ce qui constituait selon le tribunal un crime contre l'humanité (Avalon Project, 2006)). Malgré les dénonciations du contenu abominable du Stürmer par des membres du Parti nazi, Hitler a toujours soutenu Streicher en soulignant que ce type de propagande était utile pour soulever les masses à l'intellect grossier. Plus récemment, le Tribunal pénal international pour le Rwanda (TPIR) a condamné les responsables des médias rwandais durant le génocide à des peines d'emprisonnement de 35 ans et à perpétuité pour avoir encouragé les massacres. L'incitation est un crime en elle-même, mais la poursuite articula son argumentation autour d'une démonstration que l'incitation fonctionne réellement, qu'elle avait été une cause du génocide.

Cette idée, que le discours public peut directement influencer l'acteur au point de lui faire commettre des atrocités auxquelles il n'aurait autrement pas songé participer, est si largement partagée que peu de chercheurs n'ont jamais pensé à la tester réellement. Certains la mettent en doute, mais sans vraiment démontrer empiriquement sa fausseté (Waller, 2002), satisfaits d'avoir de leur côté le principe d'économie théorique voulant que ce soit l'hypothèse positive qui doive faire ses preuves (le fameux rasoir d'Occam).

Dans les deux cas, de l'Holocauste et du génocide rwandais, les médias furent utilisés intensivement. Le contenu informationnel proposé allait bien sûr dans le sens du projet gouvernemental constituant le mégacrime en question. Cependant, dans les deux cas, le public cible de l'assaut médiatique était déjà largement gagné à la cause ; l'Allemagne n'est pas devenue antisémite en 1933, et l'histoire du Rwanda est faite de conflits et de génocides. Dans la perspective poststructuraliste du discours, la relation entre le contenu médiatique (les discours au sens courant) et l'univers conceptuel servant à observer et à comprendre le monde (le discours au sens foucaldien) est symbiotique. Si on peut instrumentaliser les médias, les transformer et les intégrer au projet génocidaire par exemple, on ne peut pas en faire autant avec la culture générale, du moins c'est un programme à beaucoup plus long terme. Pour être efficace, le contenu médiatique doit s'intégrer à une culture plus large et partager le même langage.

Par ailleurs, il faut savoir différencier l'instrumentalisation des médias, qui a été évidente, par exemple, en Yougoslavie et au Rwanda, de leur effet réel sur le public cible (ce que ne font pas Kellow et Steeves, 1998). C'est une chose de décrire la prise de contrôle des 
médias et le discours public des organes de diffusion des messages de haine; c'en est toute une autre de démontrer que ce contrôle a permis aux autorités d'affecter radicalement le comportement de la population. En se penchant sur cette question avec rigueur on s'aperçoit rapidement que l'information qui permettrait cette démonstration est excessivement difficile, voire impossible à ramasser.

\section{Conclusion: quelques questions de recherche}

Ainsi, malgré plusieurs tentatives de rendre compte de divers mégacrimes, les résultats restent peu probants pour le criminologue. Le tableau ci-dessous résume brièvement les avenues empruntées jusqu'à présent.

TABLEA U 1

Conception de la normativité selon diverses explications de la participation à des mégacrimes

\begin{tabular}{lll}
\hline Explication & Normes & Problèmes \\
\hline $\begin{array}{l}\text { Abandon aux instincts } \\
\text { (classique); xénophobie, } \\
\text { agressivité naturelle } \\
\text { (évolutionniste) }\end{array}$ & $\begin{array}{l}\text { Absentes ou de force } \\
\text { insuffisante }\end{array}$ & $\begin{array}{l}\text { Les instincts sont contra- } \\
\text { dictoires; reste à expliquer } \\
\text { pourquoi certains dominent } \\
\text { à l'occasion }\end{array}$ \\
$\begin{array}{l}\text { «Diffusion» bureau- } \\
\text { cratique }\end{array}$ & $\begin{array}{l}\text { Présentes et recon- } \\
\text { nues, mais absence } \\
\text { ou diminution de la } \\
\text { responsabilité } \\
\text { individuelle }\end{array}$ & $\begin{array}{l}\text { 1) Suppose que les normes } \\
\text { existantes excluent } \\
\text { ordinairement le recours } \\
\text { à la violence }\end{array}$ \\
$\begin{array}{l}\text { Déshumanisation ou } \\
\text { «désindividualisation» }\end{array}$ & $\begin{array}{l}\text { Présentes, mais ne } \\
\text { s'appliquant pas aux } \\
\text { victimes }\end{array}$ & $\begin{array}{l}\text { 2) Suppose i) une diffé- } \\
\text { renciation des normes et } \\
\text { ii) des contextes chez les }\end{array}$ \\
$\begin{array}{l}\text { Situation de crise / } \\
\text { danger immédiat } \\
\text { (ressources, survie) }\end{array}$ & $\begin{array}{l}\text { Absolues / intransi- } \\
\text { geantes }\end{array}$ & dent à celle du chercheur \\
$\begin{array}{l}\text { Coercition (pairs, } \\
\text { ordres, processus } \\
\text { bureaucratique, l'État) }\end{array}$ & $\begin{array}{l}\text { Présentes mais } \\
\text { impossibles à respecter }\end{array}$ & \\
\hline
\end{tabular}

Plusieurs auteurs visant une explication multifactorielle (par exemple, Waller, 2002; Dutton et al., 2005) marient sans trop de souci ces explications diverses alors que certaines reposent sur des conceptions opposées de la normativité et de la relation des acteurs à leurs propres 
valeurs. Bref, beaucoup reste encore à faire. Un certain nombre d'autres considérations s'imposent également: en premier lieu, il convient de situer la question du crime de l'État non seulement dans les recherches sur l'incrimination, mais également dans les analyses contemporaines du rôle de l'État en matière de sécurité. Ce rôle est désormais fragmenté, décentralisé, de plus en plus confié à des acteurs privés individuels, communautaires ou corporatifs. Ici, la criminologie qui définit son objet par rapport à des règles issues du fonctionnement de l'État doit entièrement se réinventer. La tentation, relativement répandue, de substituer le droit international au droit national lorsque les crimes sont commis par l'État est une tentation qui se révélera sans doute peu productive à cause de sa circularité par trop évidente. La notion de crime de l'État doit sans doute aussi être revue. Malgré les distinctions comme celle qui peut être établie entre les régimes criminels et les régimes de criminels (Rosenberg, 1991) ou entre pouvoir abusif et abus de pouvoir, qui introduisent une différence entre l'action concertée et l'action d'individus déviants dans une machine étatique, il n'en reste pas moins que la confusion du concept d'État avec une entité pensante et active est peu utile. Les personnes morales que sont l'État et ses institutions et agences sont formées à la base par des individus, et l'action étatique est le fruit de leurs décisions qui certes sont à situer dans un contexte systémique, mais qui sont tout de même réfléchies au niveau personnel.

Deuxièmement, il faut désormais composer avec l'existence du Tribunal pénal international (TPI), qui semble définitivement lancé malgré ses difficultés conceptuelles, politiques et pratiques, ainsi que sa contradiction évidente avec un nombre toujours grandissant de juridictions où des mégacrimes ont été résolus de manière non pénale (en Afrique du Sud, notamment; voir Leman-Langlois, 2000; 2002; 2003 ; 2005 ; Leman-Langlois et Shearing, 2004; 2007). Le TPI replace la responsabilité de l'État vers ceux qui le dirigent; ainsi la machine étatique n'est plus qu'une collection d'actions individuelles plus ou moins réfléchies et volontaires (selon qu'on ait donné ou reçu des ordres).

Troisièmement, le mégacrime, comme nous le prouvent les attaques de Bali ou celles du 11 septembre 2001, est à la portée d'acteurs non étatiques (Leman-Langlois et Brodeur, 2005). Or, contrairement à la science politique, la criminologie est fondée sur l'étude d'acteurs à différents niveaux de pouvoir social (et surtout ceux qui sont au bas de l'échelle) et est donc, en principe, déjà mieux outillée pour comparer des dynamiques d'action à travers des contextes sociaux différents. 
Quatrièmement, la question de définir cette catégorie de crime reste entière. Les criminologues ont l'habitude de travailler sur des objets dont la définition reste chancelante, mais ceux qui sont intéressés par le processus de définition et d'incrimination y trouveront leur compte. Non pas qu'il faille raviver la sempiternelle question de l'objet de la criminologie ou des biais traditionnels qui sont à la base de sa définition, mais bien qu'une sociologie de l'incrimination a beaucoup à apprendre de phénomènes où ces définitions sont des enjeux capitaux pour de multiples acteurs sociaux.

Cinquièmement, les criminologues (surtout les étudiants) soucieux du rendement de leur travail en ce qui a trait à leur carrière devraient mieux comprendre que bien que si le passé est garant de l'avenir et qu'ainsi le Canada semble bien à l'abri du déclenchement impromptu d'un génocide, plusieurs aspects de ce type de phénomène ont effectivement des conséquences locales sur les Canadiens. Nous avons parmi nous des victimes et des coupables de génocides et autres mégacrimes. Nous envoyons nos soldats, nos policiers et nos civils dans des endroits du monde où ils seront confrontés à des mégacrimes et devront y répondre et en faire la prévention, et bien sûr y survivre eux-mêmes physiquement et psychologiquement. Au-delà de ces considérations pratiques vient également l'aspect théorique: peut-on prétendre comprendre le crime sans le mégacrime? Doit-on trouver de savantes explications pour le meurtre en oubliant les meurtres de masse extrêmes?

Pour conclure, et ici nous nous écartons de la criminologie pour faire un court détour en philosophie politique, le constat que le mégacrime est une conduite morale devrait non pas choquer et faire fuser les accusations de relativisme, voire de nihilisme, mais plutôt faire comprendre que les droits, la sécurité et la paix ne sont pas des données propres à la simple existence humaine, mais bien des produits artificiels de la volonté des citoyens, qui doivent être continuellement appréciés et protégés.

\section{Références}

Adorno, T. (1969). Éléments de l'antisémitisme. In T. Adorno \& M. Horkheimer, La dialectique de la raison (trad. française, 1974: 177-219). Paris: Gallimard. Akers, R. (1998). Social Learning and Social Structure: A General Theory of Crime and Deviance. Boston: Northeastern University Press.

Arendt, H. (1951). Le système totalitaire. Paris: Seuil, 1990.

Arendt, H. (1963). Eichmann à Jérusalem. Paris: Gallimard, 1991. 
Avalon Project (2006). Nuremberg War Crimes Trials. Yale Law School, <www. yale.edu/lawweb/avalon/imt/imt.htm>.

Bauman, Z. (1989). Modernity and the Holocaust. Ithaca (NY): Cornell University Press.

Brannigan, A. (1998). Criminology and the Holocaust: Xenophobia, Evolution, and Genocide. Crime and Delinquency, 44 (2), 257-276.

Breton, A. \& Wintrobe, R. (1982). The Logic of Bureaucratic Conduct: an Economic Analysis of Competition, Exchange and Efficiency in Private and Public Organizations. New York: Cambridge University Press.

Breton, A. \& Wintrobe, R. (1986). The Bureaucracy of Murder Revisited. Journal of Political Economy, 94 (5), 905-926.

Brodeur, J.-P. \& Ouellet, G. (2005). Qu'est-ce qu'un crime? Une réponse laïque. In Commission du droit du Canada, Qúest-ce qu'un crime? Mémoires du concours Perspectives juridiques 2002 (35-75). Québec: Les Presses de l'Université Laval.

Browning, C. (1992). Ordinary Men: Reserve Police Battalion 101 and the Final Solution in Poland. New York: HarperCollins.

Buford, B. (1991). Among the Thugs. New York: Mandarin.

Casoni, D. \& Brunet, L. (2003). Philosophie groupale et action terroriste. In D. Casoni \& L. Brunet, Comprendre l'acte terroriste(75-92). Montréal: Presses de l'Université du Québec.

Crozier, M. \& Freidberg, E. (1977). L'acteur et le système. Paris: Seuil, 1992.

Cusson, M. (2005). La délinquance, une vie choisie, entre plaisir et crime. Montréal: Hurtubise.

Day, L. E. \& Vandiver, M. (2000). Criminology and Genocide Studies: Notes on what Might Have Been and what Still Could Be. Crime, Law and Social Change, 34, 43-59.

Dutton, D., Boyanowsky, E. \& Bond, M. H. (2005). Extreme Mass Homicide: From Military Massacre to Genocide. Aggression and Violent Behavior, 10, 437-473.

Ghigilieri, M. (1999). The dark side of man: Tracing the origins of male violence. Reading, (MA): Perseus Books.

Goldhagen, D. J. (1996). Hitler's Willing Executioners. New York: Knopf.

Gourevitch, P. (1998). We Wish to Inform You that Tomorrow We Will Be Killed With Our Families. New York: Picador.

Hagan, J. (2003). Justice in the Balkans: Prosecuting War Crimes in the Hague Tribunal. Chigago: The University of Chicago Press.

Hatzfeld, J. (2003). Une saison de machettes. Paris: Seuil.

Gottfredson, M. \& Hirschi, T. (1994). Aggression. In T. Hirschi \& M. Gottfredson, The Generality of Deviance (23-45). New Brunswick (É-U): Transaction.

Israël, District Court of Jerusalem (1992). Criminal Case No. 40/61. The Attorney General of the Government of Israël v. Adolf, the Son of Adolf Karl Eichmann, Jérusalem, Trust for the Publication of the Proceedings of the Eichmann Trial, in Cooperation With the Israel State Archives and Yad Vashem, the 
Holocaust Martyrs' and Heroes' Remembrance Authority; édition critique anglaise à partir des transcriptions en hébreu, allemand et yiddish du procès de 1962.

Jenkins, R. (1996). Social Identity. Londres: Routledge.

Kellow, C. \& Steeves, L. (1998). The Role of Radio in the Rwandan Genocide. Journal of Communication, 48 (3), 107-128.

Kelman, H. \& Hamilton, V. L. (1989). Crimes of Obediance: Towards a Social Psychology of Authority and Responsibility. New Haven: Yale University Press.

Leman-Langlois, S. (2000). Mobilizing Victimization: the Construction of a Victim-Centered Approach in the South African Truth and Reconciliation Commission. Criminologie, 33 (1), 145-166.

Leman-Langlois, S. (2002). Constructing a Common Language: The Myth of Nuremberg In The Problematization of Post-Apartheid Justice. Law and Social Inquiry, 27 (1), 79-100.

Leman-Langlois, S. (2003). La mémoire et la paix, la notion de «justice postconflictuelle» dans la Commission vérité et réconciliation en Afrique du Sud. Déviance et Société, 27 (1), 43-57.

Leman-Langlois, S. (2005). Le modèle vérité et réconciliation, victimes, bourreaux et l'institutionnalisation du pardon. Informations sociales, 127, 112-121.

Leman-Langlois, S. \& Brodeur, J.-P. (2005). Terrorism Old and New. Police Practice and Research, 6 (2), 121-140.

Leman-Langlois, S. \& Shearing, C. (2004). Repairing the Future: The South African Truth and Reconciliation Commission at Work. In G. Gilligan \& J. Pratt, Crime, Truth and Justice: Official Inquiry, Discourse, Knowledge (222242). Londres: Willan.

Leman-Langlois, S. \& Shearing, C. (2007, à venir). Transition, Forgiveness and Citizenship: The South African Truth and Reconciliation Commission and the Social Construction of Forgiveness. In F. Du Bois \& A. Pedain, Justice and Reconciliation in Post-Apartheid South Africa. Cambridge: Cambridge University Press.

Mannoni, P. (2004). Les logiques du terrorisme. Paris: In Press.

Matza, D. (1964). Delinquency and Drift. New York: Wiley.

Merari, A. (1990). The Readiness to Kill and Die: Suicide terrorism in the Middle East. In W. Reich, The Origins of Terrorism (192-207). New York: Cambridge University Press.

Pape, R. (2005). Dying to Win: the Strategic Logic of Suicide Terrorism. New York: Random House.

Popper, K. (1985). The Rationality Principle. In D. Miller, Popper Selections (357-365). Princeton (New Jersey): Princeton University Press.

Rosenberg, T. (1991). The Children of Cain: Violence and the Violent in Latin America. New York: Penguin Books.

Sageman, M. (2004). Understanding Terror Networks. Philadelphia: University of Pennsylvania Press. 
Spector, M. \& Kitsuse, J. (2001). Constructing Social Problems. New Brunswick (É-U): Transaction.

Sutherland, E. \& Cressey, D. (1966). Principes de criminologie. Paris: Cujas (version originale anglaise, 1947).

Waller, J. (2002). Becoming Evil: How Ordinary People Commit Genocide and Mass Killing. Oxford: Oxford University Press. 